questionnaire (ACQ) scores of patients attending a specialist asthma clinic, as compared to objective parameters used for assessing disease severity.

Methods Measurements of IgE, Blood Eosinophils, FEV1\%, FVC\%, FEV1/FVC ratio, BMI and ACQ were recorded for patients attending an asthma clinic. BTS management step was also looked at.

Results 110 Pts mean (SD) age 52 (17), BMI 31 (8), ACQ 2.7 (1.4), Step BTS 3.5, FEV1\% 71 (22.6) FEV1/FVC 69 (15.2) were investigated. There was a strong correlation between ACQ and BMI $(r=0.244, p<0.05)$ and BTS step $(r=0.411, p=<0.05)$ but not IgE or FEV1/FVC rato. There was a weak correlation between ACO and Blood Eosinophils $(r=-0.184, p=<0.05)$. There were significantly worse ACQ scores in those with BMI $>=30 \mathrm{Kg} / \mathrm{m}^{2}(\mathrm{p}<0.05)$ and $\%$ FVC $(p<0.05)$ but no significant differences in FEV1\%, IgE, Blood Eosinophils or FEV1/FVC.

Conclusion Obesity appears to have asignificant influence on ACQ scores as a measure of asthma control and needs tobe taken into account when using this measure as an indication of severity, andformulating management plans with regards to patient care.

1. Scott S, Currie J, Albert P, Calverley P, Wilding JP. Risk of misdiagnosis, health-related quality of life, and BMI in patients who are overweight withdoctor-diagnosed asthma. Chest. 2012Mar; 141(3):616-24

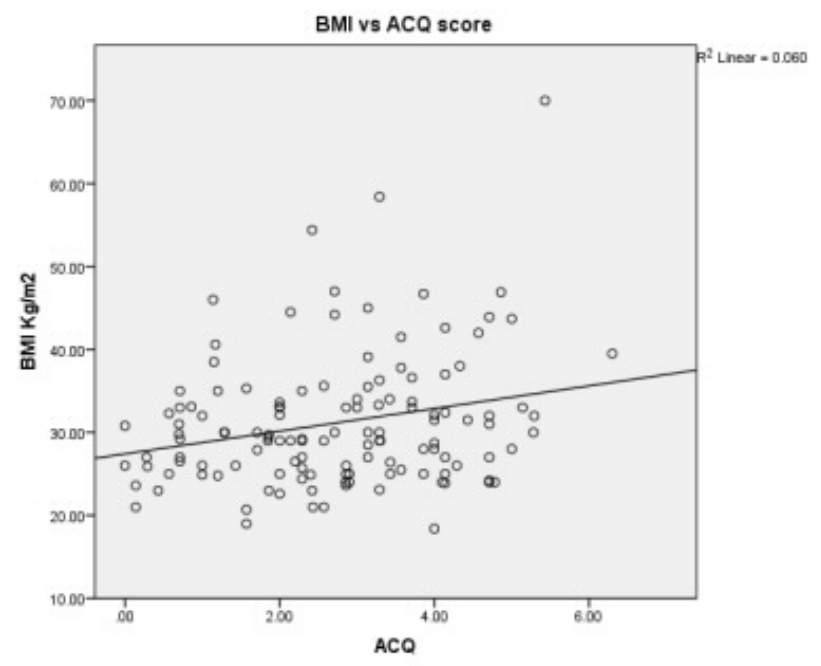

Abstract P272 Figure 1

\section{P273 ASTHMA: IS IT AS PREDICTABLE AS THE SEASONS?}

doi:10.1136/thoraxjnl-2012-202678.365

${ }^{1} \mathrm{DH}$ Thakker, ${ }^{1} \mathrm{P}$ Cullinan, ${ }^{2} \mathrm{R}$ Hubbard, ${ }^{2} \mathrm{CJ}$ Smith, ${ }^{1} \mathrm{SJ}$ MacNeill. ${ }^{1} / m p e r i a l$ College London, London, UK; '2University of Nottingham, Nottingham, UK

Background Current literature shows a distinct peak in asthma exacerbations in September among children, attributing it in part to their return to school and the spread of viral agents accompanying this. There is comparatively little recent research into the trends in adults, however. This study examines seasonal variations in asthma exacerbations in a population of adults with asthma from the community.

Methods The study population was identified from The Health Improvement Network database of anonymised GP records. Patients were between 16-40 years and had a prescription for asthma treatment during a qualification window of 1998-2000. Current analyses are restricted to patients with at least 5 years of data available pre-qualification. In this instance we defined exacerbations as acute oral prednisolone prescriptions. The daily total number of exacerbations was calculated from 01-01-1999 to 31-12-2003. To explore the potential relationship between exacerbations and comorbidities, we also explored antibiotic and antiviral prescriptions as well as treatments for seasonal allergic rhinitis in this cohort.

Results 38,439 patients with current asthma were identified. Prednisolone exacerbations decreased from the beginning of the year until summer, at which point there was a peak in June (Figure: solid line). They then increased in autumn peaking at the end of October, then increasing throughout winter. During the summer there was a similar peak observed in both prednisolone exacerbations and seasonal allergic rhinitis prescriptions in this cohort. From September through to May the trends observed in prednisolone exacerbations were similar to those seen in antiviral and antibiotic prescriptions.

Conclusions Within the seasonal trends observed, there appears to be some correlation between the summer peak in exacerbations and seasonal allergic rhinitis prescriptions. There are also similarities seen in exacerbations during the beginning and end of the year and antibiotic and antiviral prescriptions. These findings suggest that infections and seasonal allergic rhinitis might be drivers for asthma exacerbations in adults.

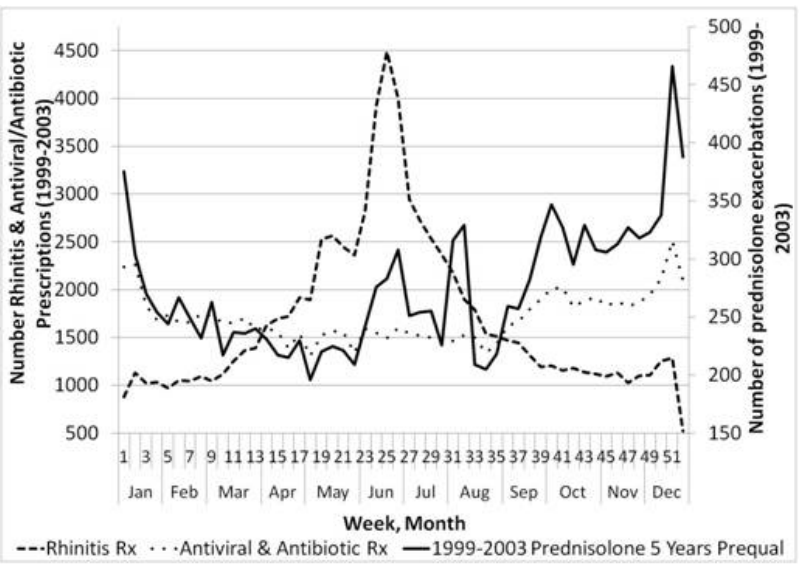

Figure: Daily total number of prednisolone exacerbations, allergic rhinitis prescriptions and antibiotic/antiviral prescriptions between 1999 and 2003

Abstract P273 Figure 1

\section{P274 DESIGNING A BEHAVIOURAL-EDUCATIONAL INTERVENTION USING INTERVENTION MAPPING TO REDUCE THE HIGH RATES OF PAEDIATRIC ASTHMA HOSPITAL ADMISSIONS IN AN INNER-CITY AREA OF BIRMINGHAM}

doi:10.1136/thoraxjnl-2012-202678.366

${ }^{1} \mathrm{~F}$ Ikram-Bashir, ${ }^{1} \mathrm{~L}$ Barrett, ${ }^{2} \mathrm{C}$ Cummins, ${ }^{3} \mathrm{H}$ Pattison. 'Birmingham Children's Hospital, Birmingham, UK; ${ }^{2}$ Birmingham \& Black Country Collaboration of Leadership for Applied Health Research and Care (BBC-CLAHRC) Theme 2, asthma project funded by the Heart of Birmingham (HOB) Trust, Birmingham, UK; ${ }^{3}$ Aston University, Birmingham, UK

Background Interventions based on empirically supported theory are effective in eliciting behaviour change (BC) (Michie \& Prestwich, 2010). We used intervention mapping to design a BC intervention to promote effective asthma management.

Method An evidence review on BC interventions for asthma was conducted; quantitative admissions data was collated; and qualitative research was used to explore family and patient experiences. These were used in the six processes of intervention mapping: needs assessment, proximal programme objective matrices, theory-based 